Check for updates

Cite this: Lab Chip, 2019, 19, 608

\title{
Latchable microfluidic valve arrays based on shape memory polymer actuators $\uparrow$
}

\author{
Bekir Aksoy, (iD $\ddagger^{\mathrm{a}}$ Nadine Besse, (D) $\ddagger^{\mathrm{a}}$ Robert Jan Boom, ${ }^{\mathrm{b}}$ Bas-Jan Hoogenberg, ${ }^{\mathrm{b}}$ \\ Marko Blom ${ }^{\mathrm{b}}$ and Herbert Shea (iD *a
}

\begin{abstract}
We report arrays of latching microfluidic valves based on shape memory polymers (SMPs), and show their applications as reagent mixers and as peristaltic pumps. The valve design takes advantage of the SMP's multiple stable shapes and over a hundred-fold stiffness change with temperature to enable a) permanent zero-power latching in either open or closed positions (>15 h), as well as b) extended cyclic operation (>3000 cycles). The moving element in the valves consists of a tri-layer with a $50 \mu \mathrm{m}$ thick central SMP layer, $25 \mu \mathrm{m}$ thick patterned carbon-silicone (CB/PDMS) heaters underneath, and a $38 \mu \mathrm{m}$ thick styrene ethylene butylene styrene (SEBS) impermeable film on top. Each valve of the array is individually addressable by synchronizing its integrated local Joule heating with a single external pressure supply. This architecture significantly reduces the device footprint and eliminates the need for multiplexing in microfluidic large scale integration $(\mathrm{mLSI})$ systems.
\end{abstract}

Received 27th September 2018,
Accepted 10th December 2018

DOI: $10.1039 / c 81 c 01024 b$

rsc.li/loc
Phase change materials (PCMs), in particular waxes and shape memory polymers (SMPs), are well suited as microfluidic valves given their inherent latching capability and their control by Joule or radiative heating. ${ }^{15-18}$ Latching valves based on paraffin wax have been extensively studied, ${ }^{18-21}$ with Yang and $\operatorname{Lin}^{18}$ being the first to propose a solution to avoid liquid contamination from direct paraffinto-liquid contact, hence allowing for cyclic operation. Their device integrates a heater below the liquid channel and separates the paraffin from the liquid using a thin compliant membrane. The main challenges for going from a single valve to large arrays are the fabrication of densely packed valves using PCMs, the integration of addressable heating elements as external stimuli, and the reduction of crossheating between neighboring valves. Hua et al. ${ }^{22}$ used carbon-loaded wax and an external computer-controlled digital projector to selectively heat each valve and pneumatic actuation to trigger their motion.

Photoresponsive polymers have enabled microfluidic valves controlled by an external light source, ${ }^{23,24}$ thus avoiding any physical contact between the controller and the valve array. The number of individually controlled valves can be increased by using an array of light sources or by micropatterned light irradiation. ${ }^{23-27}$ Although photoresponsive microvalves allow non-contact fluid manipulation, their response time is relatively slow, generally tens of seconds to completely switch.

Here, we present arrays of individually addressable, latchable, and reversible valves based on SMP actuators capable of cyclic and latching operations. Each valve can be 
selectively addressed and displaced by synchronizing the local Joule heating with a single global pneumatic air pressure. $^{28}$ The device exploits the intrinsic multistability of SMPs and over a hundred-fold stiffness change caused by a small temperature sweep through its glass transition region. By patterning stretchable carbon-silicone composite heaters with high spatial resolution on a thin SMP membrane, the selected valves can be Joule heated without altering the neighboring valves. To date, only one-shot valves using preshaped SMPs have been reported, ${ }^{17}$ despite several benefits of using solid-to-solid PCMs over liquid-to-solid ones, principally their ease of integration and their wide range of glass transition temperatures. Our approach avoids any microfluidic multiplexing strategies, replacing all external pneumatics by an electronic control board and a single pressure source. Our prototype shown in Fig. 1 has 16 valves of $3 \mathrm{~mm}$ in diameter within an active area of 15 $\mathrm{mm} \times 15 \mathrm{~mm}$. The valve array is connected by a ribbon cable to a 16 channel electrical driver board and to one external pressure supply. This SMP actuator concept is scalable to larger arrays, with a matrix of 768 actuators for haptics previously reported by our group. ${ }^{28}$

In this paper, first we explain the device operating principle and fabrication, then we report the performance of a single valve, and finally we illustrate the versatility of the SMPvalve technology in an array format by making a reagent mixer and a peristaltic pump, the two essential functional units of mLSI systems.

\section{Valve array design and operation}

The microfluidic platform, shown in Fig. 1, consists of an array of $4 \times 4$ latching valves on a $4 \mathrm{~mm}$ pitch. The main novelty lies in the active tri-layer composed of a $50 \mu \mathrm{m}$ thick shape memory polymer (SMP) membrane, with $25 \mu \mathrm{m}$ thick carbonsilicone (CB/PDMS) heaters patterned on the bottom side, and an impermeable $38 \mu \mathrm{m}$ thick styrene ethylene butylene styrene (SEBS) film on the top. This active tri-layer is sealed between a polystyrene (PS) microfluidic chip on top and a printed circuit board (PCB) on the bottom. The PCB is mounted on a polymethyl methacrylate (PMMA) pneumatic chamber. Electrical power to the heaters is routed via the PCB.

Each valve motion is selectively, independently, and electrically triggered by synchronizing the pressure from a single global pneumatic air supply with the local Joule heating of each SMP actuator. A similar actuation concept was previously reported by our group for haptic display applications, moving an array of pins that the user explored using his or her sense of fine touch. ${ }^{28,29}$ The requirements for microfluidics and haptics differ in terms of displacement, force, hermeticity, and lifetime. Key architecture differences for the SMP valves are the moving pin interface, which is replaced by a rigid microfluidic chip with an array of valve seats, and a soft SEBS film, which is used to separate the SMP membrane from the liquid. This SEBS membrane is essential to the valve operation, since it prevents performance degradation due to humidity. ${ }^{30}$

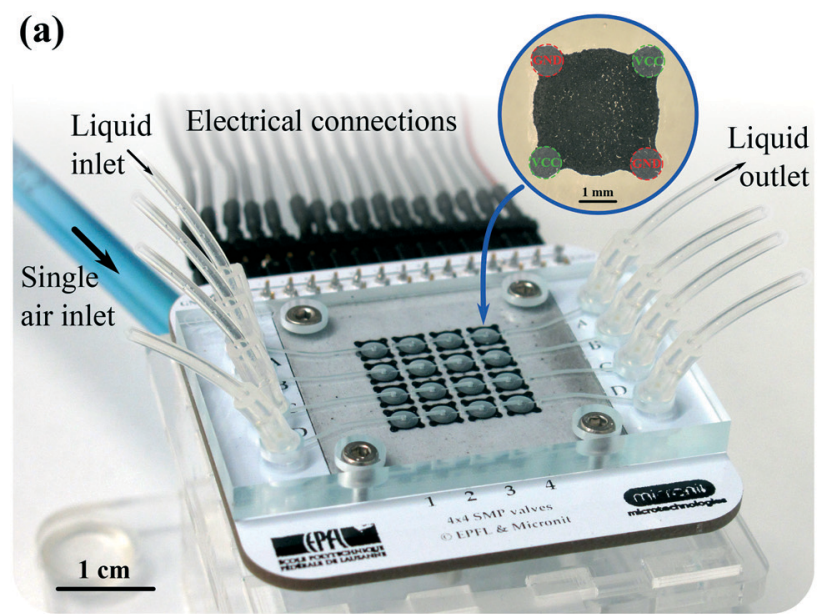

(b)

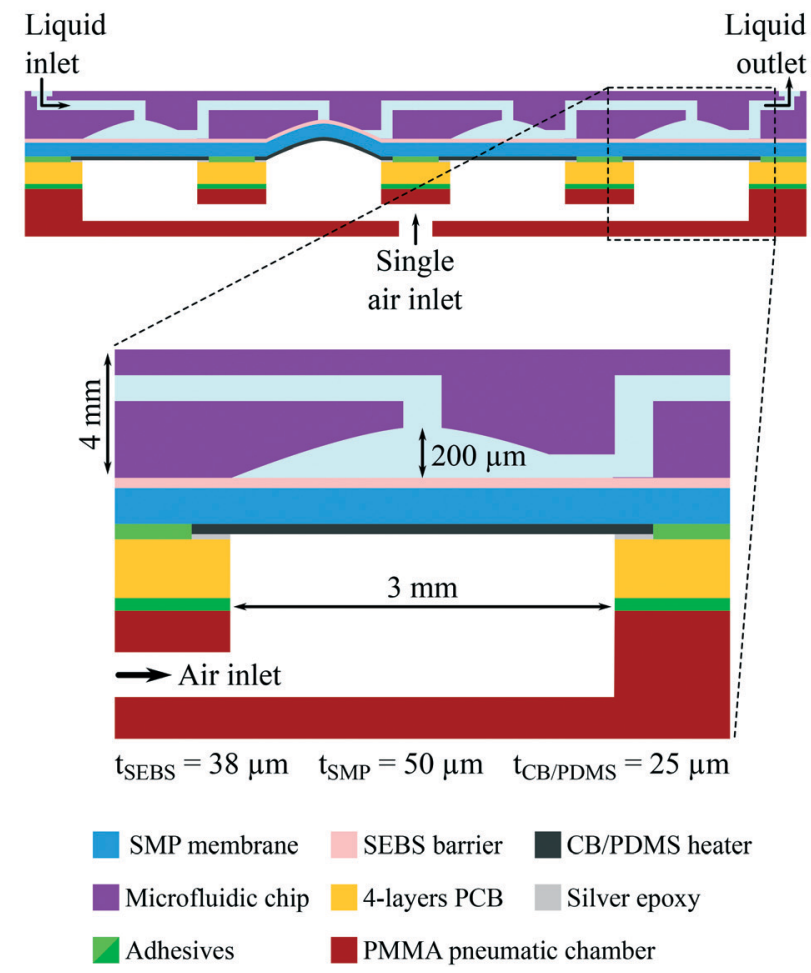

Fig. 1 Microfluidic platform with 16 latching SMP valves. (a) Complete fabricated device with an inset showing a micrograph of one heating element. (b) Schematic cross-section of one row (4 valves: 3 open and 1 closed) with a zoom on a single open SMP valve to highlight the critical dimensions.

\subsection{Valve operating principle}

The operating principle of our SMP valves exploits key intrinsic properties of SMP actuators: their over a hundred-fold change in stiffness with less than $30^{\circ} \mathrm{C}$ temperature change around the glass transition temperature, their shape programmability and recoverability, and their permanent latching capability.

A complete actuation cycle to switch a valve requires 6 consecutive steps, as illustrated in Fig. 2a:

1. The valve starts to latch in the open state. 
2. To reach the closed state, a positive air pressure of 200 mbar and a local Joule heating power of $350 \mathrm{~mW}$ (at a constant voltage of $18.8 \mathrm{~V}$ ) are applied simultaneously for $2 \mathrm{~s}$, moving the membrane up to close the valve.

3. The positive air pressure is maintained while the SMP actuator is allowed to cool for $3 \mathrm{~s}$.

4. The air pressure is removed and the valve is latched in its stable closed state.

5. To return to the open state, a negative air pressure of $100 \mathrm{mbar}$ and a local Joule heating power of $350 \mathrm{~mW}$ (at a constant voltage of $18.8 \mathrm{~V}$ ) are applied simultaneously for $2 \mathrm{~s}$. The membrane softens and is pulled down.

6. The air pressure is removed and the SMP actuator is allowed to cool for an additional $3 \mathrm{~s}$, now locked in the open state.

The cycle can then start anew.

Fig. 2b shows the dynamic mechanical analysis of single layer SMP and tri-layer membranes (DMA Q800 from TA instruments). The SMP material used here exhibits a hundredfold change in Young's modulus between its cold glassy state $\left(<40{ }^{\circ} \mathrm{C}\right)$ and its hot rubbery state $\left(>70{ }^{\circ} \mathrm{C}\right)$, with a negligible
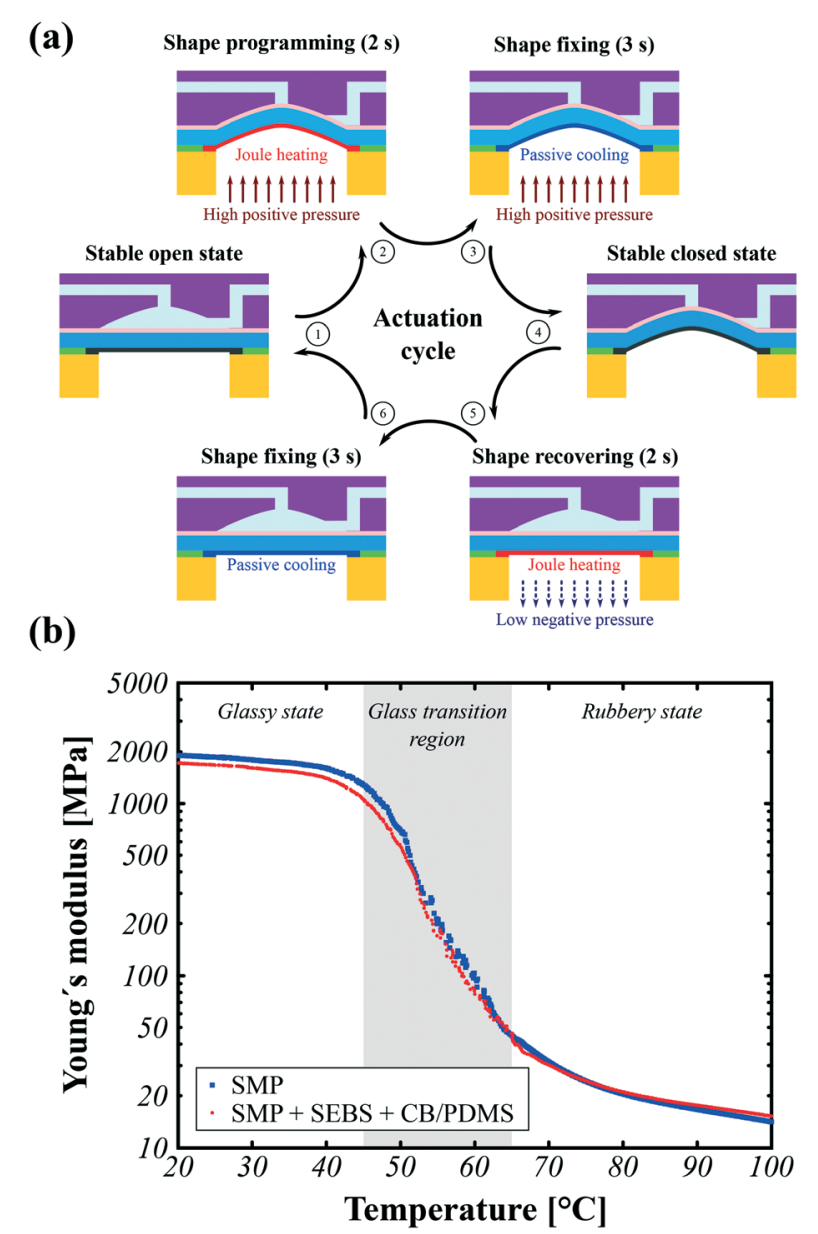

Fig. 2 Working principle of our SMP valves. (a) Schematic illustration of the complete valve actuation cycle (open $\rightarrow$ closed $\rightarrow$ open). By combining pneumatic pressure and Joule heating, each valve can be cyclically open and closed. (b) Measured Young's modulus versus temperature for the SMP material used as a single thin layer and in the tri-layer. influence from the SEBS and CB/PDMS layers. Hence, by integrating one heater per valve, it is possible to obtain a selective valve as large displacement upon external pneumatic pressure is only achieved when the valves are in the "soft" state, but negligible motion is observed for the valves in the "hard" state. Power is only needed to change states.

\subsection{Valve design and modeling}

The PS microfluidic chip and the SMP actuators were codesigned for effective valving. We chose an actuator diameter of $3 \mathrm{~mm}$, and a maximum displacement of the top of the SMP membrane of $200 \mu \mathrm{m}$, based on experimental displacements obtained with our haptic tactile pixels (taxels). ${ }^{28}$ The PS valve shape was then designed to allow for good sealing with this SMP actuator displacement, and for low enough flow impedance when the SMP actuator is flat. The main parameters to optimize are the thickness of the SMP layer and the heating profile (uniformity and temperature) of the membrane. The SMP membrane thickness influences both the actuator displacement and the power consumption. On the one hand, the thinner the SMP membrane, the larger the displacement and the shorter the switching time. On the other hand, the thicker the SMP membrane, the higher the holding force and the easier the manufacturing, but the longer the thermal switching time.

For the finite element modeling (FEM) analysis reported in Fig. 3, the heat distribution, and thus the SMP membrane stiffness, are assumed to be homogeneous over the actuated area. Hence, two main parameters influence on the valve performance: the SMP membrane thickness and the Joule heating temperature. To move, the actuation air pressure applied below the actuator should counteract the liquid pressure present above the actuator, meaning that applying simultaneously a liquid pressure of 100 mbar on top and an air pressure of 200 mbar on the bottom is equivalent to applying only $100 \mathrm{mbar}$ of actuation air pressure on the bottom. Under these conditions, FEM simulations show that the SMP membrane must be thinner than $50 \mu \mathrm{m}$ and that the heating temperature should be higher than $70{ }^{\circ} \mathrm{C}$ to operate properly. Under this $100 \mathrm{mbar}$ of liquid pressure, the valve is expected to remain closed in the cold state since the resulting displacement is negligible $(<2$ $\mu \mathrm{m})$. The heater design, shown in the inset of Fig. 1a, generating the most homogeneous heat distribution over the actuated zone was previously found to be a 4-pad circular heater with opposite pads short-circuited. ${ }^{28}$ The uniformity of the temperature is important as only the regions of the membrane that are hotter than the glass transition temperature will be significantly deformed when the air pressure is applied. The heater thickness was chosen to generate the required $350 \mathrm{~mW}$ to reach $70{ }^{\circ} \mathrm{C}$ using less than $20 \mathrm{~V}$, so as to simplify the control electronics.

\subsection{Fabrication and methods}

The fabrication process of active layers is adapted from our previously reported research. ${ }^{28,31}$ First, polyethylene terephthalate (PET) is cut to A4 size to serve as a substrate. A sacrificial layer of Teflon (100 nm thick) is blade-cast on PET to 
(a)

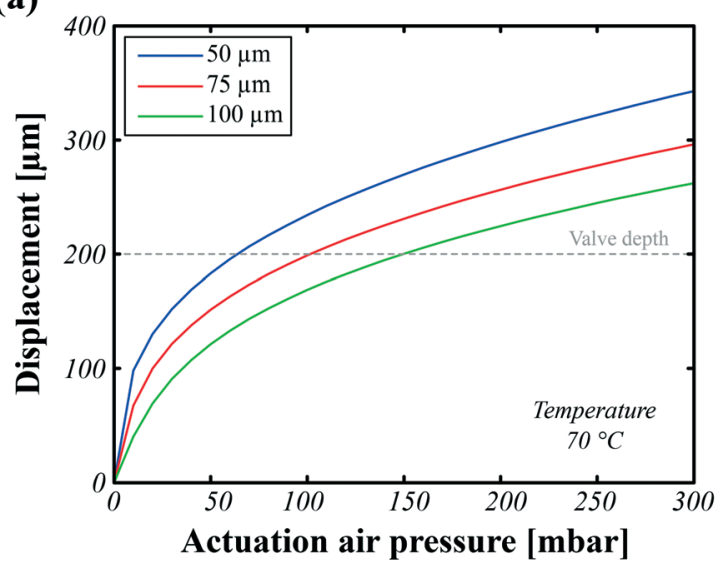

(b)

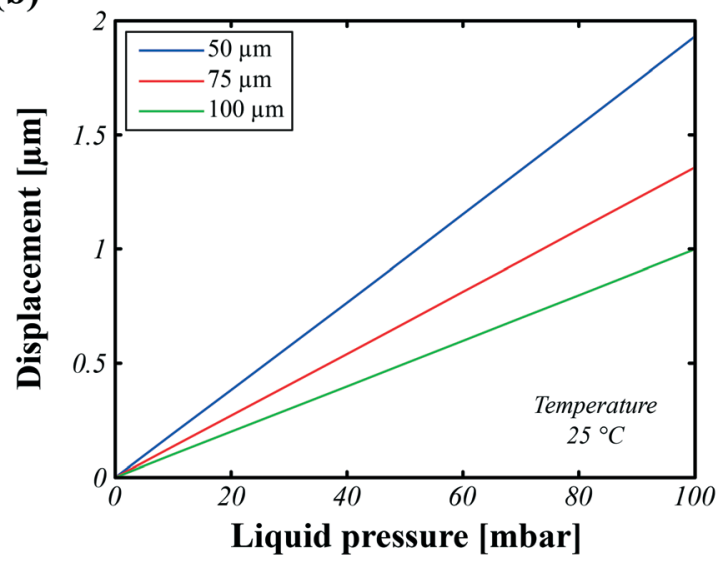

(c)

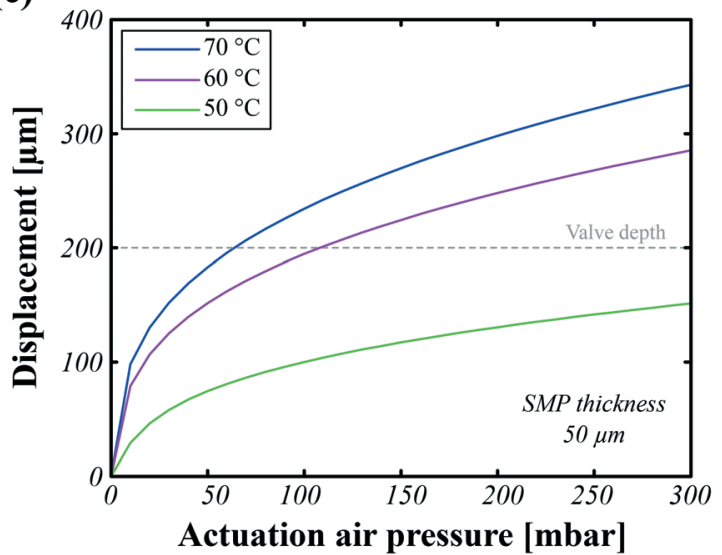

Fig. 3 Optimization of the active tri-layer thickness using FEM simulations, comparing $50 \mu \mathrm{m}, 75 \mu \mathrm{m}$, and $100 \mu \mathrm{m}$ thick SMP layers. (a) Displacement of the center of the membrane versus actuation air pressure in the hot state. (b) Displacement of the center of the membrane versus liquid pressure in the cold state, reporting how much the membrane moves due to liquid pressure when the air pressure is off. (c) Displacement of the center of a $50 \mu \mathrm{m}$ thick membrane versus actuation air pressure for several temperatures, showing larger motion when the SMP is hotter, and hence softer.

ease the removal of active layers from PET after curing. SEBS pellets (from HEXPOL TPE AB) are dissolved in toluene (ratio $1: 1)$ in a centrifugal mixer for 10 minutes. The mixture is cast on Teflon and toluene is evaporated at room temperature for $12 \mathrm{~h}$. SMP pellets (SMP MM4520 from SMP Technologies Inc.) are mixed with dimethylformamide (DMF) at a weight ratio of $1: 5$, then dissolved at $80{ }^{\circ} \mathrm{C}$ for $12 \mathrm{~h}$. This mixture is cast on top of the SEBS layer. To evaporate DMF, a programmable hot plate is used (for $4 \mathrm{~h}$ with positive and negative ramps of $60{ }^{\circ} \mathrm{C} \mathrm{h}^{-1}$ from $20{ }^{\circ} \mathrm{C}$ to $80{ }^{\circ} \mathrm{C}$ and a plateau of $4 \mathrm{~h}$ at $\left.80^{\circ} \mathrm{C}\right)$. A compliant electrode layer based on a mixture of carbon-black (Ketjenblack EC-600JD from Akzo Nobel N.V.) and polydimethylsiloxane (Silbione LSR 4305 from Elkem Silicones) is cast on SMPU using a mask for patterning. ${ }^{31}$ The stack is finally put in an oven to cure the electrode layer at $80{ }^{\circ} \mathrm{C}$ for $2 \mathrm{~h}$. This method leads to an active tri-layer with a $50 \mu \mathrm{m}$ thick SMP membrane, a $25 \mu \mathrm{m}$ thick CB/PDMS layer with an electrical conductivity of $38.3 \mathrm{~S}$ $\mathrm{m}^{-1}$, and a $38 \mu \mathrm{m}$ thick SEBS barrier. The SMP MM4520 has a glass transition temperature $T_{\mathrm{g}}$ of $45{ }^{\circ} \mathrm{C}$; other materials from the same vendor are available with a range of $T_{\mathrm{g}}$.

The active tri-layer is mechanically and electrically bonded to the PCB using acrylic adhesive with silver epoxy in the vias for the interconnects. The PMMA pneumatic chamber is attached to the bottom of the PCB using another type of acrylic adhesive (VHB4905 from 3M). Finally, this stack is hermetically sealed under pressure to the microfluidic chip at $80{ }^{\circ} \mathrm{C}$ for $2 \mathrm{~h}$.

The valves are controlled by a custom circuit that synchronizes the individual Joule heating with the common applied positive or negative air pressure. The valve characterization setup is composed of a commercial pressure regulator driving the liquid through the microfluidic chip (MFCS-EZ from Fluigent Inc.), a flow-rate sensor evaluating in real-time the liquid flow rate (FLOW UNIT M connected to FLOWBOARD from Fluigent Inc.), a platinum resistance temperature detector (RTD) sensing the liquid temperature (PPG101C1 from US Sensor Corp.), and digital multimeters measuring the heater resistance and Joule heating power (Model 2000 from Keithley Inc.). All the instruments are controlled using LabVIEW from National Instruments Corp., with data postprocessed using MATLAB from MathWorks Inc.

\section{Results and discussion}

The speed, the maximum holding pressure, and the lifetime of a single SMP valve are first reported. The mixing and pumping applications of a matrix of SMP valves are then demonstrated.

\subsection{Single valve performance}

Fig. 4 shows the cyclic and latching performance of a single SMP valve where cyclic operation is carried out for $3000 \mathrm{cy}$ cles with $5 \mathrm{~s}$ of switching between the open to the closed states and $10 \mathrm{~s}$ of latching in either state. Hence, a complete cycle lasts $30 \mathrm{~s}$. The latching performance of the valves is evaluated over $15 \mathrm{~h}$.

The actuation steps reported in Fig. $2 \mathrm{~b}$ are highlighted in the single cycle shown in Fig. 4a, in which the flow rate for a 
fixed liquid pressure is plotted as a function of time as the valve is opened and closed. A detailed graph showing the flow rate, heating power, and pump pressure with high resolution is provided as a supplementary Figure in the ESI. $\dagger$ The valve maintains a good seal for the closed state and a constant flow rate for the open state.

As discussed in the design section, obtaining a good seal in the closed state depends primarily on: the heat distribution homogeneity in the hot state to achieve complete deformation, the applied air pressure, the SMP membrane properties in the cold state for high holding pressure, and the resulting matching shapes between the deformed SMP actuator and valve chip design. The maximum holding pressure without leaking is found experimentally to be $70 \mathrm{mbar}$ to 80 mbar by incrementally increasing the fluid pressure at the closed state (see ESI $\dagger$ Fig. S1a), while simulations predicted holding pressures of over 100 mbar. This difference is probably due to an inhomogeneous temperature distribution over the SMP actuator during actuation, resulting in a locally insufficiently softened SMP, hence a smaller actuator displacement, and thus in a mismatch between the displaced SMP actuator shape and valve chip design. Fig. 4b plots the liquid

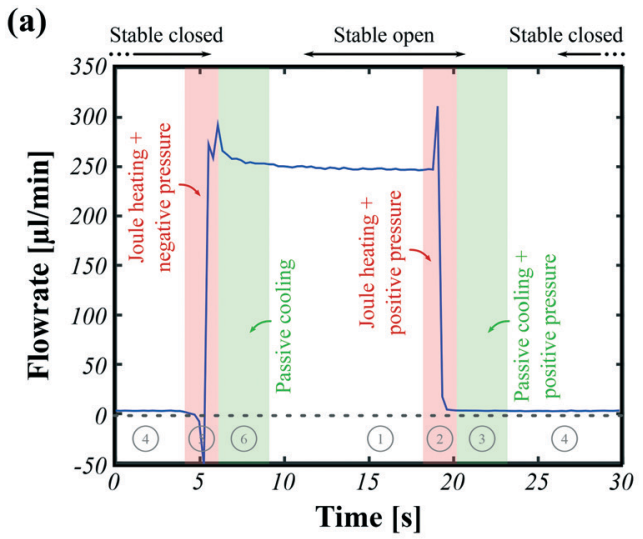

(c)

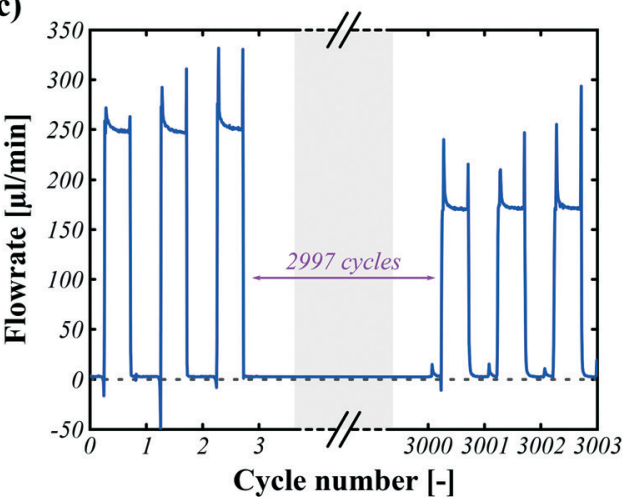

(e)

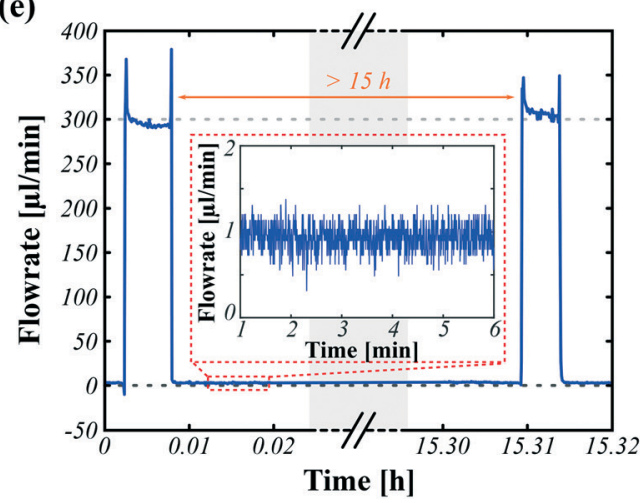

(b)

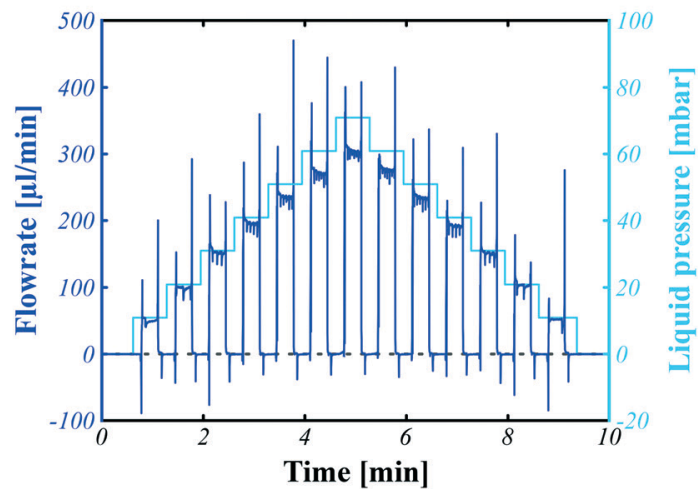

(d)

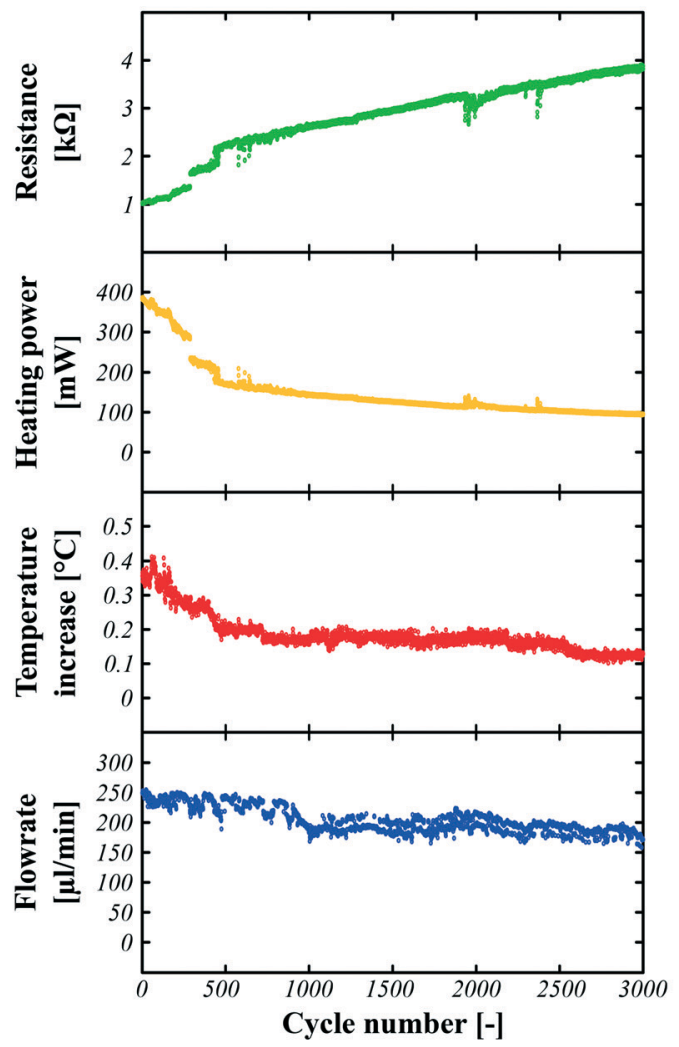

Fig. 4 Performance of a single SMP valve. (a) Flow rate versus time for one open and closed cycle with an applied liquid pressure of 50 mbar. (b) Flow rate versus time for cyclic open/closed operation while varying the pressure of the liquid. (c) Flow rate versus time for 3003 open/closed cycles showing only the first 3 and last 3 cycles. (d) Evolution over 3000 open/closed cycles of the average heater electrical resistance, Joule heating power, temperature increase in liquid, and flow rate for the valve in the open state. Because a fixed value of the heater voltage is used, the increase over time of the heater resistance directly affects the Joule heating power, temperature, and flow rate. (e) Measured flow rate versus time showing latching for over $15 \mathrm{~h}$. The leak rate in the latched closed state is shown in the inset, and the average value is $1 \pm 0.15 \mu \mathrm{lmin}{ }^{-1}$. 
flow rates as the valves are opened and closed for different liquid pressures, ranging from 10 mbar to 80 mbar with increments of 10 mbar. There is a clear proportionality between the liquid flow rate and applied liquid pressure.

At high flow rates, negative and positive spikes are observed in the flow rate when the valve switches. When the valve is switched from the open to closed state, the fluid present in the valve chamber is pushed through the outlet, causing a brief flow surge. Similarly, when the valve is opened, the membrane is pulled down and this pulls a small amount of liquid in, creating a brief backflow.

The valves are latched in both closed and open states. As shown in Fig. 4a and b, there is no flow in the latched closed state. The flow rate at the open state shows a small drift, possibly due to the time constant to fully cool the SMP membrane.

To investigate the lifetime, one randomly selected valve was operated periodically for $>3000$ cycles $(>25 \mathrm{~h})$, as reported in Fig. 4c and d. To simplify the control, a Joule heating voltage of $19.5 \mathrm{~V}$ was used in order to initially generate $375 \mathrm{~mW}$ (as the heater ages, the heating power will drop for constant voltage), the liquid pressure was set to $50 \mathrm{mbar}$, the positive air pressure to close the valve to $200 \mathrm{mbar}$, the negative air pressure to open the valve to $100 \mathrm{mbar}$, the heating duration to $2 \mathrm{~s}$, the cooling duration to $3 \mathrm{~s}$, and the latching duration to $10 \mathrm{~s}$. The complete opening and closing cycle thus lasts $30 \mathrm{~s}$. The heater electrical resistance, Joule heating power, temperature in the flow channel outlet, and liquid flow rate are continuously monitored, and their average value per cycle is plotted in Fig. $4 \mathrm{~d}$. The measurement method is further explained in the ESI. $\uparrow$ Fig. 4c captures the liquid flow rate of the first and last 3 valving cycles out of the 3003 cycles. The flow rate at the open state decreases by $30 \%$ over the thousands of cycles. This is mainly related to a slow degradation (resistance increase) of the carbon-based electrodes, as reported by de Saint-Aubin et $a .^{32}$ For simplicity of the setup, a constant voltage scheme was used for Joule heating rather than a constant power circuit. As the resistance of the heater increases with the number of deformation cycles, the heater power at fixed voltage decreases, leading to less heating, and hence less softening of the SMP membrane, which thus no longer fully opens, leading to a reduced liquid flow rate. The degradation of the electrode occurs much faster than the deterioration of the SMP. After 3000 cycles of switching, the SMP retains its mechanical properties and can still latch the valve at the closed state up to $70 \mathrm{mbar}$ liquid pressure. The speed of electrode degradation depends on the deformation the electrode undergoes at each cycle. Lower deformation provides a longer lifetime.

As microfluidic devices are often used with living cells, ${ }^{33,34}$ the valve must not meaningfully change the temperature of the liquid flowing through it. ${ }^{35}$ A temperature sensor was inserted in the tubing at the microfluidic channel outlet, which is located at $30 \mathrm{~mm}$ from the activated valve (this was as close to the valve as the sensor could be positioned). As seen in Fig. 4d, the temperature increase in the liquid is less than $0.4^{\circ} \mathrm{C}$. As shown in the ESI, $\dagger$ the temperature fluctuation of the liquid depends on the flow rate, being higher and shorter for fast flow rates and smaller and longer for slow flow rates. In any circumstances, despite heating the active layer at $>70{ }^{\circ} \mathrm{C}$, temperature fluctuations at the sensor of $<1$ ${ }^{\circ} \mathrm{C}$ are observed.

Unlike PDMS-based microfluidic valves, ${ }^{12,13}$ SMP-based valves can intrinsically latch, allowing stable prolonged continuous use in the open or closed state. To demonstrate this latching capability, a valve is operated for several open/closed cycles at 50 mbar of liquid pressure, then latched at the closed state for $15 \mathrm{~h}$, and finally it is intentionally reopened and cycled to control its behavior, keeping the same liquid pressure for the entire experiment.

Fig. 4e plots the flow rate as a function of time. The flow rates at open states before and after latching are near $300 \mu \mathrm{l}$ $\min ^{-1}$. A close-up view of the flow rate at the closed-latched state is shown in the inset of Fig. 4e, which is $1 \pm 0.15 \mu \mathrm{l}$ $\mathrm{min}^{-1}$. Normally-open (NO) valves are studied in this paper, meaning that the valve is open at rest when the active layer is undeformed. The critical state here is the closed-latched state since the active layer has to preserve its deformed shape under liquid pressure. As reported in the ESI, $\dagger$ if the liquid pressure at the closed-latched state is higher than the internal holding pressure of the membrane, the liquid will flow. In contrast, in the open-latched state, the liquid pressure always helps to keep the valve open.

\subsection{First application: reagent mixer}

The microfluidic platform with 16 valves shown in Fig. 1b has been attached to a different PS microfluidic channel top chip to accommodate 8 valves ( 4 inlets and 4 outlets) with a laminar flow mixing channel in the middle. This design allows simultaneous control of several valves and observation of the mixing of the liquid.

We first used the device to reorder the inputs, as an illustration of the ability to control multiple valves simultaneously. For ease of visualization, we used different color food dyes dissolved in water. In addition to distilled water (W) as a rinsing liquid, the following colors are used: blue (B), yellow (Y), and red (R). First, as shown in Fig. 5a, the valves are actuated to re-order the color sequence at the device outlets without mixing them. In the inlet channels reagents are ordered as BYR. By switching simultaneously one inlet valve and one outlet valve, a single color is transferred at a time. When color transfer is finished, both open inlet and outlet valves are closed, both valves located at the extremities of the device are opened, and the mixing channel is rinsed to the waste container. This procedure is applied to all inlet colors so as to obtain RYB at the device outlet. A real-time video is provided in the ESI, $\dagger$ with configurations after each color transfer step shown in Fig. 5a.

Secondly, as the name implies, the mixer is also used to mix the three inlet colors. For this, two inlet valves and one 
(a)
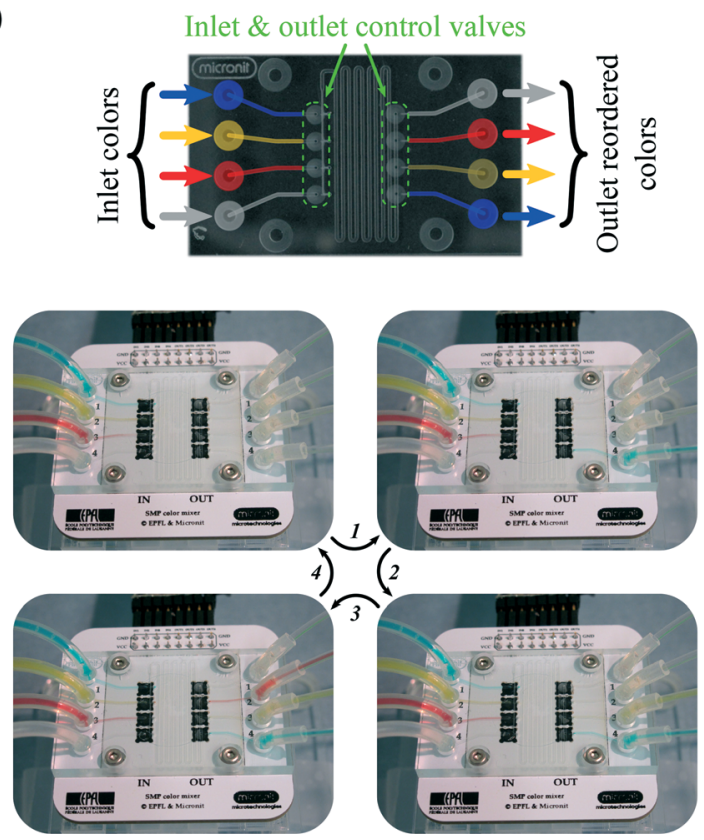

(b)

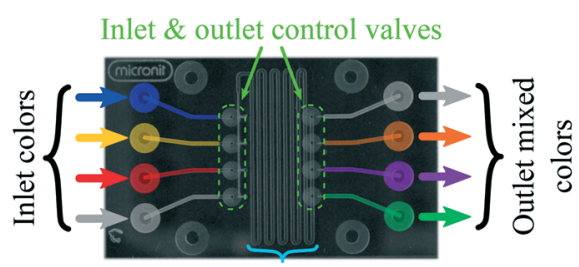

Mixing channel

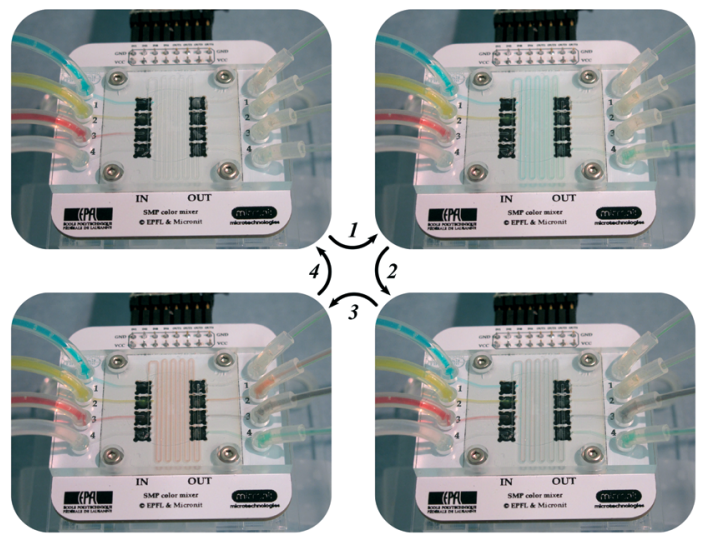

Fig. 5 Reordering and mixing colors. (a) Pictures of the successive reordering of blue, yellow, and red. (b) Pictures of the successive mixing of blue + yellow, of blue + red, and of yellow + red. Supplementary videos in the ESI† show real-time mixing and reordering.

outlet valve are simultaneously actuated, resulting in three sets of two mixed colors at the outlet: green $(\mathbf{G}=\mathbf{B}+\mathbf{Y})$, violet $(\mathbf{V}=\mathbf{B}+\mathbf{R})$, and orange $(\mathbf{O}=\mathbf{Y}+\mathbf{R})$ colors. Here, the inlet channel reagents ordered as BYR are mixed as OVG at the device outlet. A real-time captured video is provided in the ESI, $\uparrow$ with configurations after each color mixing step shown in Fig. 5b. Although the applied liquid pressure is the same for all inlets, a variation in the flow rate between channels may occur if either the displacement of each SMP actuator or the hydraulic resistance of each tubing slightly varies.

In this mixing application, both the latching and cyclic capabilities of our valves are beneficial to minimize power consumption and device footprint (since only one pneumatic control line is used). This application shows that a set of SMP valves can be simultaneously actuated. To investigate the heating of the entire chip after extended continuous use, all the valves on the mixing chip were simultaneously actuated for $80 \mathrm{~min}$ with an average power of $350 \mathrm{~mW}$ each. As reported in the ESI, $\dagger$ the temperature of the chip increased from $26.2^{\circ} \mathrm{C}$ to $28.8^{\circ} \mathrm{C}$, a moderate increase that can be accommodated. The most suitable use for our device is application where extended latching operations are desired. No power is consumed when latched in the open or closed state, resulting in no heating.

\subsection{Second application: peristaltic pump}

Traditional PDMS-based peristaltic pumps require at least three pneumatic supplies to actuate each valve in sequence. ${ }^{8,36-38}$ Thanks to the intrinsic latching properties of SMPs, a single pressure supply is sufficient for peristaltic motion.

To make the pump, we used one row of 4 SMP valves, out of a matrix of 16, connected as shown in Fig. 6a, which also shows the sequential actuation cycle implemented to push the liquid from left to right. By opening and closing the valves in sequence, the liquid is pulled into and pushed out of the valves to create a net flow. The actuation frequency is limited by the heating and cooling time of each valve (approximately $2.5 \mathrm{~s}$ ). Fig. $6 \mathrm{~b}$ plots the instantaneous flow rate as a function of time. The repeating peristaltic pump cycle has three large positive peaks corresponding to the closing of the first three valves and one negative peak when opening the fourth valve.

During peristaltic motion neither the inlet nor outlet is pressurized: both sides are at atmospheric pressure. The only driving force for fluid motion is the opening and closing of the valves. When a valve is switched from open to closed, the fluid in this chamber exits through either the inlet or outlet. For valves \#2, \#3, and \#4, closing creates a forward flow (at the outlet) because the valves on the inlet of each valve are closed and the valves on the outlet side are open. For valve \#1, however, this process creates a backward flow, meaning the fluid in this chamber exits from the inlet channel. This backward motion is not seen in the flow-rate graph because the flow-rate sensor is placed at the outlet of valve \#4. The volume that is transferred with the peristaltic motion is shown in Fig. 6b. As depicted from this graph, the transferred volume has upward and downward steps. The average volume of upward strokes is $1.87 \pm 0.36 \mu \mathrm{l}$ (there are 3 per pump cycle) and downward strokes is $0.52 \pm 0.16 \mu$ l (only 1 per pump cycle).

As proposed by Chuang et al. ${ }^{36}$ sequential timing can be optimized to improve peristaltic pump performance. 
(a)

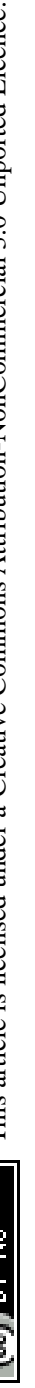

Moreover, to increase the mean flow rate, larger valves could be used, or additional valves could be implemented, a straightforward step with our approach since several sets of 4 valves could be actuated simultaneously without the need for additional pressure supplies.

\subsection{Scaling considerations}

The work shown here is a proof of principle with 16 valves, of dimensions larger than typical microfluidic valves. We chose the $3 \mathrm{~mm}$ diameter valves for compatibility with our earlier work on the $24 \times 32$ array of SMP actuators for haptics, ${ }^{28}$ in which we used row/column addressing to control 768 actuators with 56 control lines. We discuss below some key aspects for scaling down the size and for scaling up the number of valves. To provide some quantified results, we use the example of scaling the diameter down from $3 \mathrm{~mm}$ to $1 \mathrm{~mm}$ and scaling up the number of valves from 16 to 256 .

1. Electrical addressing of individual valves
Joule heating is used to locally soften the SMP membrane using integrated compliant heaters. All electrical connections to these heaters are through a single PCB. When the number of valves is increased from $16(4 \times 4)$ to $256(16 \times 16)$, row/column addressing can be used to reduce the number of control lines, then only $32(16+16)$ control lines will be sufficient to actuate 256 SMP valves.

\section{Size scaling of the valve's dimensions}

For reciprocating valves, the active tri-layer needs to be designed with a free displacement greater than the valve depth to fully block the flow. In the devices reported here, a net pressure of $100 \mathrm{mbar}$ is enough to completely close a 200 $\mu \mathrm{m}$ deep valve with a diameter of $3 \mathrm{~mm}$. The parameters that most affect the pressure needed to switch the SMP valves are: the valve depth, the actuator diameter, and the mechanical properties of the SMP membrane. To investigate the effects of these parameters on the required net pressure, FEM simulations, reported in the ESI, $\dagger$ were carried out to evaluate the net pressure required to close the valve as a function of the actuator diameter, for different valve depths. The SMP 
thickness and its mechanical properties are taken to be constant for these simulations. Reducing the actuator diameter exponentially increases the pressure required, while reducing the valve depth calls for lower required pressures. In other words, the footprint of the SMP valves can be reduced as long as the valve depth is decreased along with diameter reduction. As an example, 25 mbar of pressure can close a valve having a dimeter of $3 \mathrm{~mm}$ and a depth of $100 \mu \mathrm{m}$. However, a valve with a similar depth but with a diameter of $1 \mathrm{~mm}$ requires 775 mbar of net pressure to fully close. If we reduce the depth of this valve from $100 \mu \mathrm{m}$ to $50 \mu \mathrm{m}$ (keeping the same diameter of $1 \mathrm{~mm}$ ), then the net pressure needed drops to 225 mbar. If soft lithography methods are used for the SMP material, the dimensions of the valves can be further reduced to $100 \mu \mathrm{m} \times 100 \mu \mathrm{m} \times 10 \mu \mathrm{m}$ (typical dimensions for PDMS based valves). ${ }^{8}$

3. Heating requirements and thermal actuation speed

We reported here a circular SMP valve with a diameter of $3 \mathrm{~mm}$ (area of $7 \mathrm{~mm}^{2}$ ) that requires $350 \mathrm{~mW}$ of power to heat above the glass transition temperature. When this diameter is reduced to $1 \mathrm{~mm}\left(0.8 \mathrm{~mm}^{2}\right.$ area), only $39 \mathrm{~mW}$ is needed (9 times less). This significantly reduces the contribution of individual SMP valves to the temperature increase of the entire chip. The thermal time constant is proportional to the actuator size. The smaller the SMP valve, the faster the actuation speed. The SMP valves scale down favorably in terms of power requirement and thermal speed.

4. Fabrication related challenges

Fabrication techniques play a role in scaling. For example, we use stencil printing to pattern the CB/PDMS electrode on the SMP layer and the feature size of printed electrodes is $500 \mu \mathrm{m}$. This introduces a practical limitation on the size scaling of electrodes. Using soft lithography or other methods, the fabrication of microfluidic components with much smaller sizes is achievable. ${ }^{8}$

\section{Conclusions}

A novel microfluidic valve concept based on SMP actuators, enabling both permanent latching $(>15 \mathrm{~h})$ and extended cycling (>3000 cycles) has been described and demonstrated. The major innovations are the selective patterning of stretchable CB/PDMS heaters directly on the SMP membrane, the active layer protection from the liquid with a thin SEBS impermeable barrier, and the synchronization of each actuator local Joule heating with a global pneumatic air supply. They enable downscaling of the device footprint by eliminating the need for multiplexing and by replacing the pressure controllers with electronic drivers to individually address each valve. A reagent mixer and a peristaltic pump have been reported as demonstrators, an important step towards a simple and compact mLSI system based uniquely on SMP-based latching valves. Moreover, the small temperature increase $\left(<{ }^{\circ} \mathrm{C}\right)$ in the flowing liquid opens interesting prospects in biological applications, ${ }^{39,40}$ notably for organ-on-chips, drug delivery, and reagent mixers.

\section{Conflicts of interest}

There are no conflicts to declare.

\section{Acknowledgements}

This work was supported by the Swiss National Science Foundation, Grant number 200020-165993. We thank O. Gudozhnik and S. Rosset at the EPFL for helpful discussions.

\section{References}

1 K. Eyer, P. Kuhn, C. Hanke and P. S. Dittrich, Lab Chip, 2012, 12, 765-772.

2 R. N. Zare and S. Kim, Annu. Rev. Biomed. Eng., 2010, 12, 187-201.

3 W. H. Grover, R. H. C. Ivester, E. C. Jensen and R. A. Mathies, Lab Chip, 2006, 6, 623-631.

4 E. C. Jensen, A. M. Stockton, T. N. Chiesl, J. Kim, A. Bera and R. A. Mathies, Lab Chip, 2013, 13, 288-296.

5 J. Melin and S. R. Quake, Annu. Rev. Biophys. Biomol. Struct., 2007, 36, 213-231.

6 Z. H. Wang, M. C. Kim, M. Marquez and T. Thorsen, Lab Chip, 2007, 7, 740-745.

7 S. J. Maerkl and S. R. Quake, Science, 2007, 315, 233-237.

8 M. A. Unger, H. P. Chou, T. Thorsen, A. Scherer and S. R. Quake, Science, 2000, 288, 113-116.

9 T. Thorsen, S. J. Maerkl and S. R. Quake, Science, 2002, 298, 580-584.

10 L. M. Fidalgo and S. J. Maerkl, Lab Chip, 2011, 11, 1612-1619.

11 S. J. Maerkl and S. R. Quake, Proc. Natl. Acad. Sci. U. S. A., 2009, 106, 18650-18655.

12 S. Haeberle and R. Zengerle, Lab Chip, 2007, 7, 1094-1110.

13 D. Mark, S. Haeberle, G. Roth, F. von Stetten and R. Zengerle, Chem. Soc. Rev., 2010, 39, 1153-1182.

14 D. W. Lee and Y. H. Cho, Lab Chip, 2009, 9, 1681-1686.

15 A. Richter, D. Kuckling, S. Howitz, T. Gehring and K. F. Arndt, J. Microelectromech. Syst., 2003, 12, 748-753.

16 E. Wilhelm, C. Richter and B. E. Rapp, Sens. Actuators, A, 2018, 271, 303-347.

17 H. Takehara, C. Y. Jiang, K. Uto, M. Ebara, T. Aoyagi and T. Ichiki, Appl. Phys. Express, 2013, 6, 037201.

18 B. Z. Yang and Q. Lin, J. Microelectromech. Syst., 2009, 18, 860-867.

19 R. H. Liu, J. Bonanno, J. N. Yang, R. Lenigk and P. Grodzinski, Sens. Actuators, B, 2004, 98, 328-336.

20 R. Pal, M. Yang, B. N. Johnson, D. T. Burke and M. A. Burns, Anal. Chem., 2004, 76, 3740-3748.

21 K. W. Oh, K. Namkoong and P. Chinsung, Micro Total Anal. Syst., 2005, 1, 554-556.

22 Z. Hua, R. Pal, O. Srivannavit, M. A. Burns and E. Gulari, Lab Chip, 2008, 8, 488-491.

23 S. Sugiura, A. Szilagyi, K. Sumaru, K. Hattori, T. Takagi, G. Filipcsei, M. Zrinyi and T. Kanamori, Lab Chip, 2009, 9, 196-198.

24 S. R. Sershen, G. A. Mensing, M. Ng, N. J. Halas, D. J. Beebe and J. L. West, Adv. Mater., 2005, 17, 1366-1368. 
25 C. Delaney, P. McCluskey, S. Coleman, J. Whyte, N. Kent and D. Diamond, Lab Chip, 2017, 17, 2013-2021.

26 S. Coleman, J. ter Schiphorst, A. Ben Azouz, S. Bakker, A. P. H. J. Schenning and D. Diamond, Sens. Actuators, B, 2017, 245, 81-86.

27 S. Sugiura, K. Sumaru, K. Ohi, K. Hiroki, T. Takagi and T. Kanamori, Sens. Actuators, A, 2007, 140, 176-184.

28 N. Besse, S. Rosset, J. J. Zarate and H. Shea, Adv. Mater. Technol., 2017, 2, 1700102.

29 N. Besse, S. Rosset, J. J. Zarate, E. Ferrari, L. Brayda and H. Shea, IEEE Trans. Haptics., 2018, 11, 30-38.

30 B. Yang, W. M. Huang, C. Li, C. M. Lee and L. Li, Smart Mater. Struct., 2004, 13, 191-195.

31 S. Rosset, O. A. Araromi, S. Schlatter and H. R. Shea, J. Visualized Exp., 2016, e53423.

32 C. A. de Saint-Aubin, S. Rosset, S. Schlatter and H. Shea, Smart Mater. Struct., 2018, 27, 074002.
33 H. J. Kim, D. Huh, G. Hamilton and D. E. Ingber, Lab Chip, 2012, 12, 2165-2174.

34 J. D. Caplin, N. G. Granados, M. R. James, R. Montazami and N. Hashemi, Adv. Healthcare Mater., 2015, 4, 1426-1450.

35 Y. Reissis, E. Garcia-Gareta, M. Korda, G. W. Blunn and J. Hua, Stem Cell Res. Ther., 2013, 4, 139.

36 H. S. Chuang, A. M. Amin, S. T. Wereley, M. Thottethodi, T. N. Vijay Kumar and S. C. Jacobson, Proceedings of the 12th international conference on miniaturized systems for chemistry and life sciences, 2008.

37 H. Y. Lai and A. Folch, Lab Chip, 2011, 11, 336-342.

38 D. Kim, W. S. Lee, S. Shin, H. S. Rho, J. Dai, J. Y. Yun and J. W. Hong, Sci. Adv. Mater., 2014, 6, 2428-2434.

39 S. N. Bhatia and D. E. Ingber, Nat. Biotechnol., 2014, 32, 760-772.

40 E. K. Sackmann, A. L. Fulton and D. J. Beebe, Nature, 2014, 507, 181-189. 